\title{
Tabooed Universal Characteristic Length and Misled Boiling Heat Transfer Research
}

\author{
Irakli G. Shekriladze \\ Department of Hydro-Engineering of Georgian Technical University, 77 Kostava Street, Tbilisi 0175, Georgia
}

\begin{abstract}
The paper presents the next step within multiyear fruitless efforts of the author to overcome the absurd situation in boiling heat transfer research. The focus is made on the problem of the characteristic length of the process most clearly exhibiting the consequences of half a century ignoring the basic MTD (Model "theater of director"), the UC (Universal correlation) and some other boiling fundamentals. Echoing control of boiling heat transfer by nucleation, the MTD-UC identifies universal characteristic length, the AER (Average effective radius) of nucleation sites, equally workable at the macro- and microscale. Inefficiency of the generally accepted, so called MTA (theater of actors) is particularly pronounced just in the confusion with the characteristic length. Traditional and potential candidates, departure diameter of vapor bubble and transverse internal size of the channel hardly can be adjusted to independence of developed boiling HTC on mass acceleration, subcooling, liquid convection and the heating surface geometry. At the same time, even such a problem has not prevented many authors to develop tens or even hundreds of helpless MTA-based correlations. The ignoring the MTD-UC-AER has also led to the incompleteness of the standard boiling heat transfer experiment, which is usually done without studying nucleation sites (there are available only very few comprehensive experimental works including the data on the AER). The only exception was made for the problem of boiling heat transfer enhancement: over the past decades enhanced boiling surfaces were developed in direct accordance with the principle defined by the MTD-UC (just through the AER). Another thing is that the basic role of the MTD-UC-AER in substantial progress of the relevant R\&D activities passed over in silence in the corresponding publications. Enviable unity and coherence of heat transfer community in preventing real scientific debate on the problem is also remarked.
\end{abstract}

Key words: Nucleation, effective radius, characteristic length, boiling heat transfer.

\section{Nomenclature}

$\mathrm{C}_{\mathrm{p}} \quad$ Heat capacity of liquid phase, $\mathrm{J} /\left(\mathrm{kg} \cdot{ }^{\circ} \mathrm{C}\right)$

$\mathrm{T}_{\mathrm{s}} \quad$ Temperature of saturation, $\mathrm{K}$

h Heat transfer coefficient (HTC), W/ $\left(\mathrm{m}^{2} \cdot{ }^{\circ} \mathrm{C}\right)$

$\mathrm{k}$ Thermal conductivity of liquid phase, $\mathrm{W} /\left(\mathrm{m} \cdot{ }^{\circ} \mathrm{C}\right)$

q Heat flux, $\mathrm{W} / \mathrm{m}^{2}$

$\mathrm{r} \quad$ Heat of evaporation, $\mathrm{J} / \mathrm{kg}$

$v \quad$ Kinematic viscosity of liquid phase, $\mathrm{m}^{2} / \mathrm{s}$

$\rho \quad$ Density of liquid phase, $\mathrm{kg} / \mathrm{m}^{3}$

$\rho_{g} \quad$ Density of vapor phase, $\mathrm{kg} / \mathrm{m}^{3}$

$\rho_{0} \quad$ Average effective radius of nucleation sites, $\mathrm{m}$

$\sigma \quad$ Surface tension, $\mathrm{N} / \mathrm{m}$

\section{Introduction}

According to the MTD (Model "theatre of a director") [1-3], the uniqueness of the boiling

Corresponding author: Irakli G. Shekriladze, professor, research field: heat transfer and hydrodynamics. phenomenon is related to the control of convective heat transfer by nucleation through multiple short-run launches of different cooling mechanisms by onset of vapor bubble growth at nucleation sites. Developed boiling heat transfer turned out to be controlled regardless of the composition and inputs of involved individual cooling mechanisms. Fundamental character of the MTD is confirmed by the UC (Universal correlation) describing the experimental data on boiling heat transfer of all groups of liquids, including liquid metals and cryogens, during pool and channel flow boiling of saturated or subcooled liquid, including mini- and microchannels, without matching different constants and powers to different surface-liquid combinations [2, 4-8].

Comprehended the controlling role of nucleation, the MTD uniquely determines the AER (Average 
effective radius) of nucleation sites as the characteristic length of boiling heat transfer. The MTD also includes well controlled by the same nucleation PEGB (Pumping effect of growing bubble) [1, 3], MLE (Microlayer evaporation) [9] and MMP (Micro-membrane pumping effect) [10].

Control of heat transfer by the nucleation sites, via the characteristic length of the order of $10^{-5} \mathrm{~m}$, it determines independence of heat transfer law on the geometrics of the heating surface and creates universal context for interpretation and analysis of the entire range of developed boiling heat transfer processes in macro- and microsystems [11]. In order to analyze the diversity of boiling heat transfer curves different from the basic developed boiling curve, including boiling hysteresis, the MTD is supplemented by so-called MFC (Multi-factoring concept) [12].

At the same time, despite the obvious fundamental importance, the MTD-UC, the PEGB and others were subjected to general neglect during half a century. The UC, never challenged central result of boiling heat transfer studies, was first confirmed by independent studies only at 47 th year of publishing [4]. However, even in such an exclusive event, using wide but not comprehensive experimental database, the picture was drawn of competition between the UC and the two later "most advanced" correlations.

Suffice it to extend the database on helium, liquid metals, the heating surfaces with significantly different nucleation sites, the "competing" with the UC equations immediately become helpless. Besides, it is not by tens of percent error in predicting HTC, but at times, and an order of magnitude, turning into the firm evidence of complete fiasco of long-term extensive MTA-based research [13].

The main social obligation of the scientific community, continuous expertise and synthesis of research results and ensuring students and professionals on the reliably proven advanced knowledge, it is ignored in the field of boiling heat transfer for decades, by providing monopoly position in university textbooks and reference books for the certain outdated research direction.

In this regard, it is particularly illustrative the key article [5], claiming to the scientific breakthrough: unprecedented universal correlation of developed boiling heat transfer. The article was published in Int. J. HMT 33 years ago.

Assuming that earlier publications were difficult to reach, in the light of the above social commitment, boiling research community simply was obliged, as a minimum, to refute timely the above high claim. Despite this, the article not only did not get a decent estimate, but it has never even been cited by any boiling heat transfer researcher.

Here a challenging question naturally arises: doesn't reflect this fact, along with the reluctance to accept a breakthrough in order to preserve the above monopolistic positions, an inability to challenge the declared high claim?

Unfortunately, the relevance of such a reasoning is supported by numerous other well-documented facts.

The latter refers to the ignoring of numerous calls of the author for scientific debate, complete disregard of severe critical assessments given by him in the letters to the editors, including those unanswered, conference presentations and papers, scientific articles and reviews, decades of blocking of relevant submissions for keynote papers by the Regional Scientific Committee, failed intention to organize panel discussion on IHTC-14, unprecedented rejection of the letter to the editors (Int. J. Thermal Sciences, 2011), rejection of the proposals for much-needed discussions by the 8 th (2012) and 9th (2015) Int. Conferences on Boiling and Condensation Heat Transfer and IHTC-15 (2014).

\section{The AER and Correlation of Experimental Data on Developed Boiling HTC}

To see how unambiguously and invariably the AER can be acknowledged as a characteristic length of boiling heat transfer we should consider a number of fairly well-known facts. 
Let's start with the great discovery by Moore and Mesler (1981) of local temperature pulsations of the heating surface synchronous with a bubble formation and departure cycle. Fundamental outcome of this and further experimental studies established the coincidence of the maximum local superheat of heating surface and the start of strong cooling effect with the onset of bubble growth (Fig. 1., points a and c).

The maximum local superheat of the heating surface and the launch of powerful cooling mechanisms, primarily, the PEGB, also the MLE and the MMP, turn out to be dependent on the nucleation, i.e., the onset of a bubble growth. At the same time, the onset of a bubble growth takes place at the instant of the average temperature of the meniscus of critical size overcomes the temperature of thermodynamic equilibrium in the system nucleus-liquid-site. Within the MTD, just this size, determining maximum local superheat launching a bubble growth and cooling mechanisms, it is called as "effective radius" of the nucleation site. In the framework of the MTD, the AER, the average for the numerous roughly similar sites, turns out to be the universal characteristic length of developed boiling heat transfer, the single parameter of the heating surface impacting HTC.

During developed boiling, according to the MTD, the AER is fully determined by geometrics of nucleation sites, regardless of heat flux, saturation pressure and physical parameters of boiling substance. The AER turns roughly constant equal to $5 \mu \mathrm{m}$ on the commercial surfaces. In general case the AER can be dependent on additional factors that leads to diversity of boiling heat transfer curves, including boiling hysteresis. In more detail it can be seen in the works cited, for example, in the review [6].

These circumstances already possible to consider as unambiguous proof of AER as the characteristic length of boiling heat transfer. However, for greater cogency, let's see some more other evidences. First of all, let's demonstrate control of heat transfer exactly by nucleation, regardless of the contributions of different cooling mechanisms.

In boiling of saturated single-component liquid one can distinguish four cooling mechanisms. Among them only MLE [9] is linked to immediate evaporation on boiling surface (Fig. 2a). Other three mechanisms, bubbling [14] (Fig. 2b), the PEGB or jet-like [1] (Fig. 2c) and the MMP [10] (Fig. 2f), are linked to liquid phase convection.

With another approach number of cooling mechanisms during saturated boiling could be even greater.

For instance, cooling mechanisms through pushing the liquid by growing bubble, through displacement of overheated liquid layer (transient conduction dominated model) or through drift liquid current subsequent to detached bubble sometimes are thought to be separate mechanisms. Here they are regarded as being separate stages of action of the bubbling mechanism.

Subcooling puts in operation two additional cooling mechanisms: heat pipe-like (evaporation-condensation) (Fig. 2d), being an extra version of MLE, and BCD (Bubble collapse-driven) mechanism (quasi-cavitational) [16] (Fig. 2e) associated with the collapse of a bubble on the surface under the influence of influx of highly subcooled liquid.

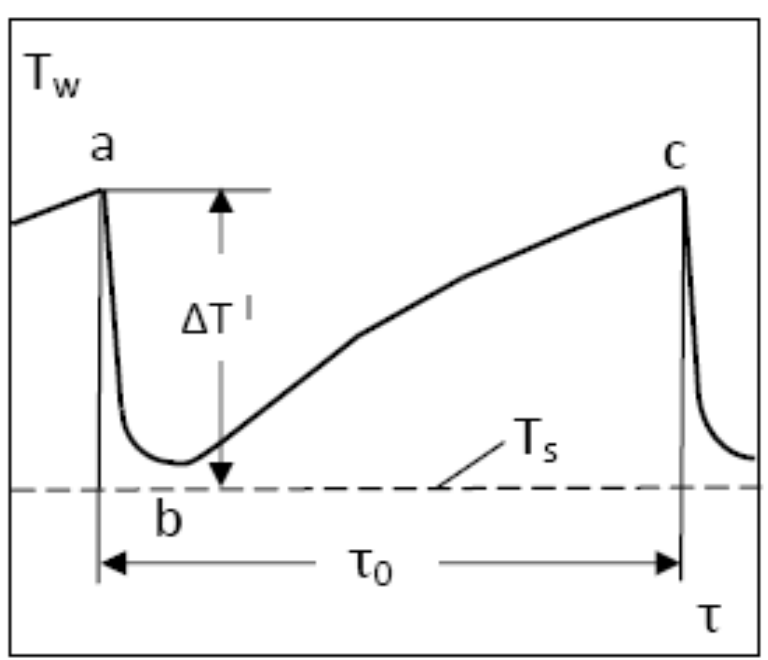

Fig. 1 Typical cycle of local temperature pulsation of heating surface. 


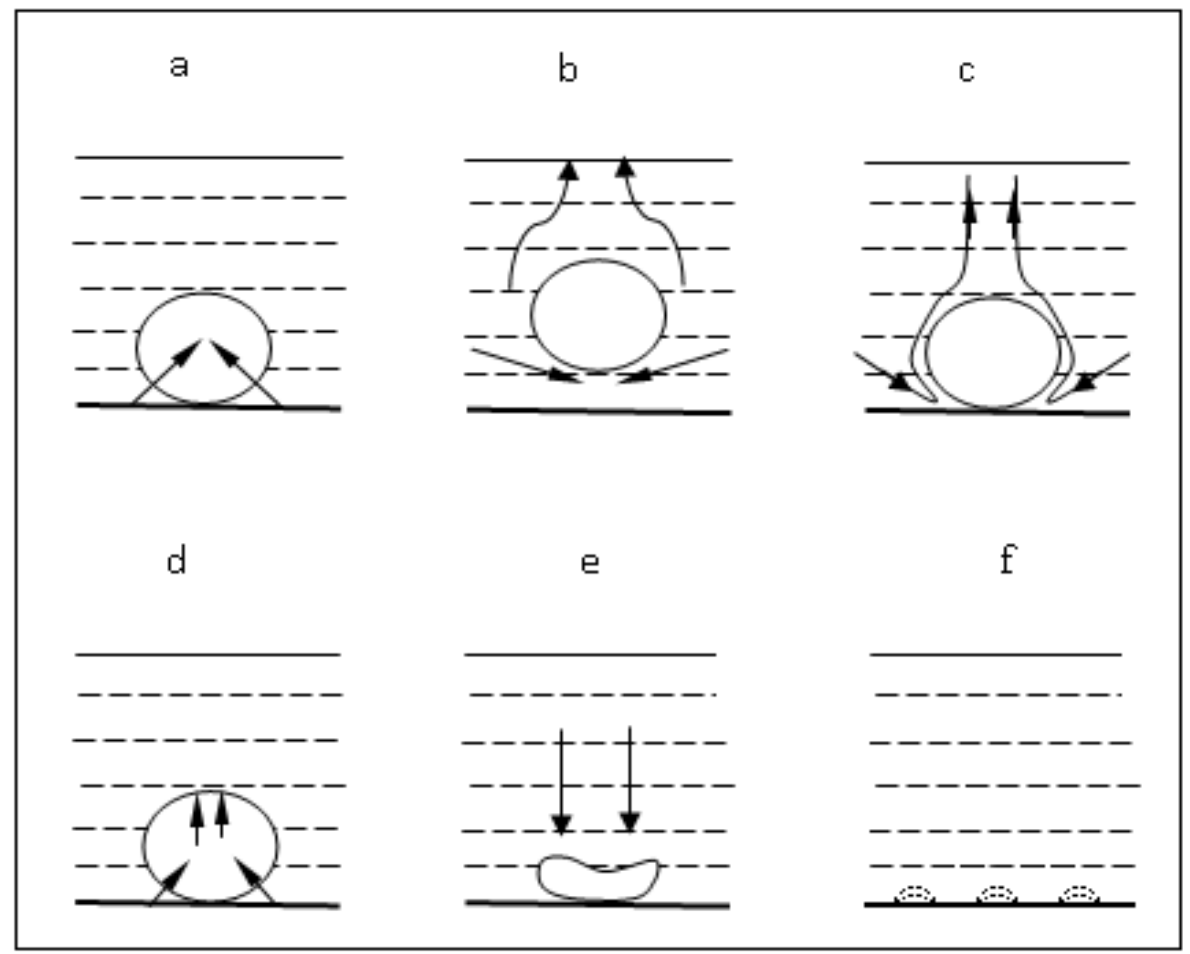

Fig. 2 Schematics of cooling mechanisms: a - MLE; b-bubbling; $c$ - PEGB; $d$ - heat pipe-like; e - BCD; f - MMP.

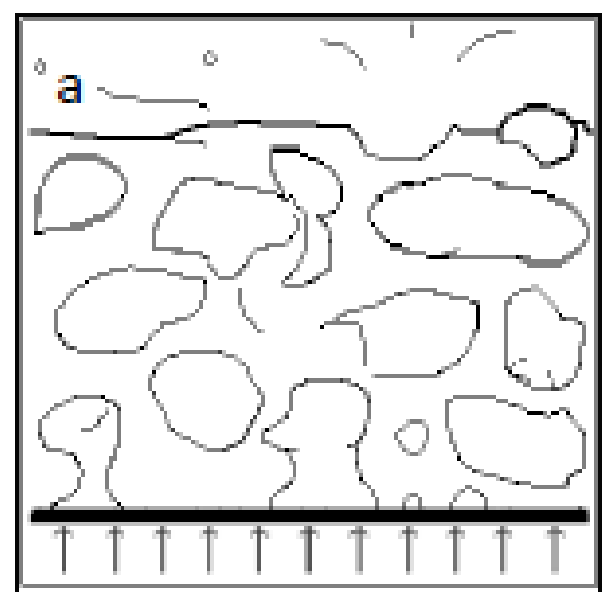

b

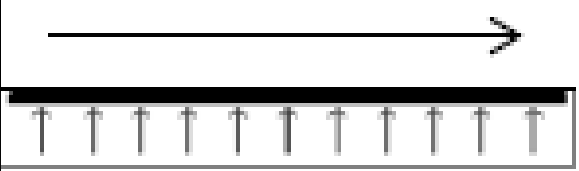

Fig. 3 Boiling according to (a) Jacob and (b) Peng and Wang.

By this goal, let's compare the two cases of boiling heat transfer shown in Fig. 3.

The first is the most familiar, "classical" pool boiling of water with bubbles growing, detaching and rising, the most widely studied since the pioneering works of Jacob and coworkers [14] and representing the basis for the description of traditional boiling heat transfer model, the MTA (theater of actors), based on the identification of the main cooling mechanism (or mechanisms) and evaluation of its intensity. The same process has led to identification of detachment diameter as the characteristic length.

From the standpoint of the MTA, in terms of intensities of involved individual cooling mechanisms, the first process drastically differs from the second one - surface boiling of deeply subcooled water flowing in the channel with internal diameter $5 \mathrm{~mm}$ [17]. It is no accident that, at high subcooling, high-speed videography with a resolution of order of a millimeter in general has not recorded any bubbles: we face the 
process with countless microscopic bubbles, collapsing right after birth even without detachment, with huge density of nucleation sites.

There is no doubt that, in these two cases, diverse individual cooling mechanisms contribute in heat transfer quite differently. Moreover, according to [16], the $\mathrm{BCD}$, which is generally absent in the first process, is quite weighty in the second one. And most importantly: despite of critical difference in the composition and intensities of cooling mechanisms, these two processes reproduce the same developed boiling heat transfer curve at equal saturation pressure.
The universality of developed boiling heat transfer law, regardless to fundamental changes in the composition and contributions of different individual cooling mechanisms, it clearly proves control of heat transfer by nucleation. In other words, it is highlighted another unambiguous proof of AER as the characteristic length of boiling heat transfer.

Next, let's demonstrate the same through correlation of experimental data on developed boiling heat transfer by the UC. For this we present the UC in the dimensional form showing the effect of separate parameters on the HTC:

$$
h=0.88 \cdot 10^{-2} q^{0.7} \rho_{0}^{0.4} r^{0.325} k^{0.3} C_{p}^{0.25} \rho^{0.25} \rho_{g}^{0.2} \sigma^{-0.45} T_{s}^{-0.45} v^{-0.25}
$$

As follows from (1), except the main regime parameter (heat flux), the AEP $\left(\rho_{0}\right)$ is one of the most influential factors on HTC. In addition, it is the only parameter of the heating surface that can be changed to enhance boiling heat transfer. Dependence of HTC on the AER can be presented in the following form:

$$
\frac{h}{A}=\rho_{o}^{0.4}
$$

where,

$$
A=0.88 \cdot 10^{-2} q^{0.7} r^{0.325} k^{0.3} C_{p}^{0.25} \rho^{0.25} \rho_{g}^{0.2} \sigma^{-0.45} T_{s}^{-0.45} v^{-0.25}
$$

Next, let's present correlation of existing experimental data on HTC by the UC in the coordinates of the equation (2) in the realistic limits of change of the AER $1.0-100.0 \mu \mathrm{m}$.

In Fig. 4, the area 1 corresponds to the experimental data on boiling HTC on commercial surfaces the AER of which was roughly equated to $5 \mu \mathrm{m}$ based on some indirect data (25 liquids [2, 6, 13], 55 liquids [4]). Further this evaluation of the AER was supported by experimental data on boiling of water on copper surfaces accompanied by investigation of the nucleation sites [18], also corresponding to the area 1. The studies cover water, organic liquids, liquid metals, refrigerants and cryogens. The UC is the only correlation describing boiling HTC of all groups of liquids.

The area 2 corresponds to the experimental data on boiling HTC of sodium on the artificial heating surface with the AER equal to $50 \mu \mathrm{m}$ [19]. The area 3 corresponds to the experimental data on boiling HTC of the refrigerants $\mathrm{R} 12$ and $\mathrm{R} 22$ on the artificial heating surface with the AER equal to $86 \mu \mathrm{m}$ [20]. These studies, along with above investigation by Shoukri and Judd, belong to a few number of comprehensive experimental works including the data on the nucleation sites.

Obviously, the correlations not including the AER (i.e., all of the many known correlations, except the UC) cannot describe the data presented in Fig. 1 in a unified manner. By the way, during boiling of sodium at $\rho_{0}=50 \mu \mathrm{m}$ HTC is 2.5 times higher than on commercial surface, other conditions are the same. The same enhancement factor for the refrigerants is 3.1.

Quite clearly emerges also a universal role of the AER regardless of the scale of the process [11].

Summing up, it is hoped, we clearly demonstrated that the AER is the real universal characteristic length of the boiling heat transfer. We also need to add a trivial conclusion, the correlation not containing the actual characteristic length, cannot give fairly complete 


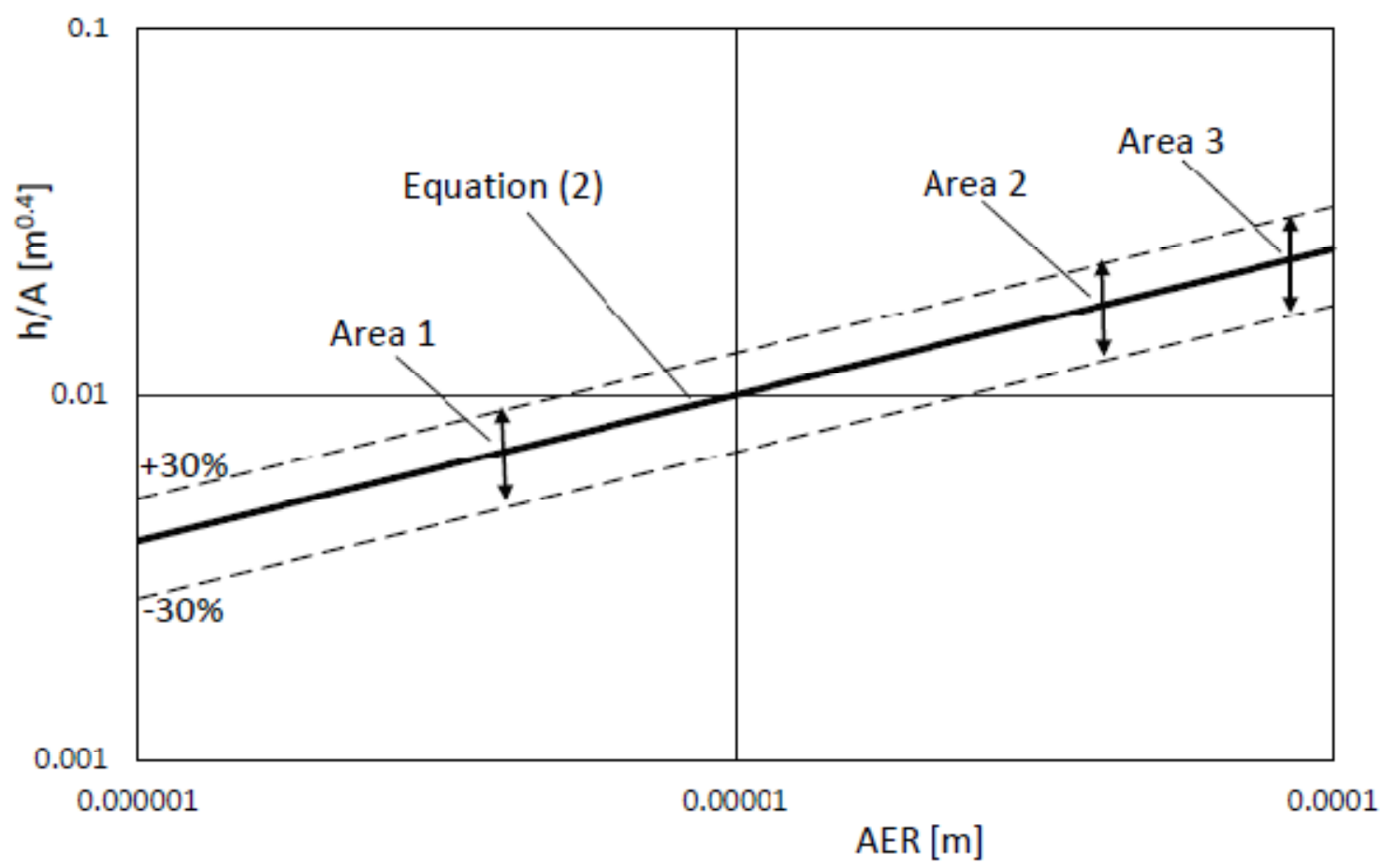

Fig. 4 Correlation of experimental data on developed boiling HTC by the UC in the coordinates of the equation (2): Area 1 commercial heating surfaces $\left(\rho_{0} \approx 5 \mu \mathrm{m}\right)$; Area 2 - developed boiling of sodium on the artificial heating surface $\left(\rho_{0}=50 \mu \mathrm{m}\right)$; Area 3 - developed boiling of the refrigerants R 12 and R 22 on the artificial heating surface $\left(\rho_{0}=86 \mu \mathrm{m}\right)$.

description of heat transfer process.

Apparently, the latter circumstance requires an expansion of the circle the possible reasons that caused misleading of boiling heat transfer research through total neglect of the MTD-UC-AER. If the above we placed the emphasis on providing a monopoly position for a certain outdated research direction, now we have to take into account also that timely introduction to the scientific turn the MTD-UC-AER would deprive the possibility of infinite development the new helpless correlations. As the need for such "theorizing" has appeared surprisingly significant, the circle of potential sufferers from recognizing the MTD-UC-AER could be much broader.

\section{The AER and Incompleteness of the Standard Boiling Heat Transfer Experiment}

Ignoring the MTD-UC-AER led to incompleteness of the standard experiment in boiling heat transfer, which is usually performed without studying nucleation sites (above we already have noted very few comprehensive experimental works including the data on AER). In other words, the experimenters do not measure just the single parameter of the heating surface that strongly impacts HTC.

If you try to invent an analogy to this situation, we could talk about the experiment on the hydrodynamics of the channel flow without measuring the cross-sectional dimensions of the channel. If such an absurd is difficult even to imagine in hydrodynamics, in boiling heat transfer it is practiced for decades.

Here should be noted also that in boiling heat transfer the situation is partly mitigated by frequent use of commercial heating surfaces (as a rule, rolled tubes), which fortunately turn out to be characterized by roughly uniform AER approximately equal to $5 \mu \mathrm{m}$. The latter circumstance, of course, makes it easier to verify the UC, but did not alter the substance of the above hiding the basic role of the MTD-UC-AER in boiling heat transfer enhancement.

The MTD incorporates so-called one-parameter model of heating surface containing unlimited number 
of similar nucleation sites (with the same effective radius), regardless of whether the site is operating or potential. It is also assumed that the AER is only a function of the geometrics the nucleation site. As abundance of similar nucleation sites always provides the "reserve" of potential sites with the same effective radius, the AER becomes a single parameter of the heating surface affecting HTC.

As shown by the correlation of the broad experimental data by the UC, such simplest idealization of heating surface turns out to be relevant in relation to developed boiling heat transfer on commercial heating surfaces and some artificial surfaces with roughly identical nucleation sites.

At the same time, there exist heating surfaces with much complex distribution of nucleation sites, providing dependence of the AER on the parameters not only the heating surface, but boiling medium and heat flux as well. Just such heating surfaces lead to diverse boiling curves, including drastically different slopes or boiling hysteresis. In order to analyze wide spectra of specific boiling heat transfer processes at macro-and microscale the MTD is supplemented by so-called MFC.

Comprehensive experimental investigation and analysis of boiling heat transfer processes is impossible without involving the real characteristic length of heating surfaces through studying nucleation sites. Ignoring this fundamental fact has brought the theory and experiment in a historical deadlock. Persistent avoiding by heat transfer community the real debate on the issue decisively contributes to this.

It still remains as the primary task physical modelling of developed boiling on one-parameter heating surfaces. We are speaking about heating surfaces with abundant identical artificial nucleation sites, for example, in the form of cylindrical recesses, providing the AER equal to the radius of the cylinder at any value of heat flux and any parameter of boiling medium, including wetting angle. Such experiments should cover boiling heat transfer of various liquids in a wide range of saturation pressure for different values of the AER in the range $1.0-100.0 \mu \mathrm{m}$.

Similar experiments will allow to establish the basic curve of developed boiling heat transfer, determine the exact values of coefficients and exponents of the UC, which, hopefully, will turn to be identical for different combinations liquid - surface and saturation parameters (judging by the above results of description by the UC of extensive experimental data on boiling on commercial surfaces).

Similar basic boiling curve will allow to analyze the spectra of different specific boiling curves, including the disclosure of such interesting phenomenon as the diversity of the slope of boiling curve and its relation to the distribution of nucleation sites by the effective radius.

Another important direction of boiling heat transfer studies should be based on the development of specific methods and tools for investigation of natural nucleation sites of the heating surfaces. The development of this direction requires to achieve a qualitatively new level of boiling heat transfer experiment, to what the research community turned to be unprepared.

That is why a pioneering study Shoukri and Judd (1979), just laid the foundation of this trend, has not received further development. As shown by the past decades, the boiling heat transfer research community is still not matured for this task.

In any case, the decisive role of the nucleation sites in heat transfer, displayed by the real universal characteristic length, the AER, it lies in the nature of the boiling phenomenon and ignoring this fact excludes the successful solution of the problem.

\section{The AER and Enhancement of Boiling Heat Transfer}

The line of development of high-efficient boiling surfaces presents a fortunate exception in the context of implementation of the MTD.

Along with describing developed boiling heat 
transfer, the MTD-UC uncovered the ways of heat transfer enhancement. In terms of the physics of the phenomenon, according to the MTD, there should serve as the main tool reducing the superheat triggering the growth of a vapor bubble, i.e., reducing the maximum superheat in the heating surface temperature cycle shown in Fig. 1. As this superheat mainly depends on the AER and surface tension, the MTD-UC offers two concrete ways of heat transfer enhancement: artificial enlargement of the AER and artificial decrease of surface tension.

In this context, it is rather convenient the presentation of the MTD in the form of equation (1), which clearly reveals that just the above two parameters influence heat transfer in the greatest extent, much more greatly than heat capacity, viscosity and thermal conduction of liquid phase. By the way, the latter is one more manifestation of controlling role of nucleation in boiling heat transfer.

It should be emphasized that the MTD has a "monopoly" only in terms of identifying the real effect of the characteristic length of boiling heat transfer (the AER). With regard to the surface tension, a similar effect is already reflected in the early correlations [21-24], though not because of the accounting the controlling role of nucleation, but resulting from use of a bubble departure diameter as the characteristic length.

In terms of technological feasibility, intensification through the AER has no alternative (the second path long stays at the stage of experimentation). For this reason, in recent decades, developers of advanced boiling surfaces were forced to follow the MTD-UC-AER, achieving significant advancement of the relevant branch of technology.

Based on so-called one-parameter model of boiling surface the MTD-UC determines the basic principle of boiling heat transfer enhancement through creation of numerous high-sized stable artificial nucleation sites with minimum worsening of thermal conductance of boiling surface, including the establishment of the quantitative frames of achievable results (HTC $\mathrm{AER}^{0.4}$ ). Besides, as Eq. (2) is valid just in the case when large sites are formed without worsening of thermal conductance of boiling surface, the UC determines the highest achievable effect.

In this context it is reasonable to return to discussion of correlation of experimental data presented in Fig. 4.

The part of these experiments just is targeted at heat transfer enhancement (areas 2 and 3). The correlation quantitatively verifies predicted by Eq. (1) heat transfer enhancement law. Real enhancement factor, as compared with commercial surfaces, in these experiments achieved around 3 in the case of refrigerants and 2.5 in the case of sodium, in full accordance with the UC. These experiments just represent the example of creation nucleation sites with large AER without worsening of thermal conductance of heating surface.

At the same time such a worsening not always may be avoided during creation of large sites. For instance, creation of porous layers always is connected with increase of thermal resistance of heating surface. Besides, negative role of additional thermal resistance becomes more tangible with increase of heat flux. This is why in experiments on enhanced boiling surfaces heat transfer enhancement factor reduces at high fluxes.

In this context the principle of minimum worsening of thermal conductance of heating surface through creation of artificial sites still remains topical through development of enhanced boiling surfaces.

However, all the above circumstances have not prevented complete silencing the MTD-UC-AER.

The role of the boiling fundamentals turned out to be hidden by a rather simple way: the important issues to specify the ideological and methodological bases of development the enhanced boiling surfaces and analyze the results in the same basic framework, they are generally avoided in the publications. You will not find a single publication, correctly indicating relying on the MTD-UC and pointing out that the creation of 
coatings or other surface structures with large nucleation sites is nothing but an increase the AER, i.e., the real universal characteristic length of boiling heat transfer.

A recent survey [25] may serve as an example of such an approach.

\section{The Problem of Characteristic Length and Boiling Heat Transfer Reviews}

This is a separate issue, reflection or rather avoiding the problem of characteristic length in the modern reviews on boiling heat transfer.

In $2000 \mathrm{~s}$, an attempt was undertaken to summarize development of boiling heat transfer theory per the XX century [26, 27], of course, in the tradition of complete disregard of the MTD-UC. According to the review, among numerous correlations, the two (Rohsenow and Pioro) can be regarded as "more accurate".

These correlations fit the experimental data on boiling heat transfer of various liquids (except liquid metals and cryogens) using different constants and powers for different surface-liquid combinations. In fact, these correlations represent the series of numerous purely empirical equations, written in non-dimensional form and uncovering once again the fundamental limitations of the MTA-based analysis. The problem of the characteristic length was not analyzed at all, except for the note that so-called capillary constant of the liquid, assumed to be proportional to the vapor bubble departure diameter, "is used in many practical non-dimensional correlations for the HTC as a characteristic dimension for nucleate pool-boiling in the Nusselt number" [26].

It was enough to see by the simplest way to the role of gravity in this length, the failure of all the correlations based on it would become immediately obvious. However, apparently, the analysis of the physical soundness of boiling heat transfer correlations was not topical in the review.

Here it is worth paying attention to a rather strange tradition originating from the work [28].
On the one hand, in this article and the review as well, starting with the title and continuing by detailed consideration of the various aspects of operation of the nucleation sites, including appropriate analytical relationships, priority attention is emphasized to the role of the heating surface in boiling heat transfer.

On the other hand, finally, the recommended relations for HTC do not include any specific parameter of the heating surface or nucleation sites.

In contrast to it, introducing the simplest idealization of the heating surface and using classical equation of Griffith and Wallis [29], the MTD reveals the universal characteristic length of boiling heat transfer just linked to the nucleation sites and leads to the UC describing developed boiling HTC of all groups of liquids.

By the way, MTD-UC was published before the work Mikic and Rohsenow [28]. In addition, UC has not undergone any upgrade since 1966.

The review [26, 27] was subjected to profound criticism from the standpoint of the MTD-UC in the letter to the editors which remained unanswered [30].

It would seem that such a public scientific defeat in the Int. J. Heat Mass Transfer was to protect the MTD-UC from ignoring in the following scientific reviews. However, it appeared only seemed.

For instance, based on analysis of the recent experimental, analytical, and numerical work into single bubble heat transfer, quite comprehensive review of boiling heat transfer mechanisms established that "transient conduction and microconvection are found to be the dominant" [31].

In other words, the review confirmed quite thoroughly the MTD, describing boiling heat transfer as an alternation of heat accumulation by transient conduction in the boundary area surface-liquid, up to achievement by nucleus the critical size, just equal to the characteristic length, and subsequent launching the PEGB, i.e., microconvection primarily focusing the accumulated heat to the surface of the growing bubble.

One need only look at the analytical solution of the MTD (the first approximation) and the scheme of the 
PEGB, to be convinced of this $[1-3,5,6]$.

Unfortunately, the survey strictly adheres to the tradition of ignoring the MTD-UC. As a result, the real main beneficiary of the study is not even mentioned.

With regard to the characteristic length, it is also of interest the study [32], aimed at shaping fundamental perspective for scale effects on flow boiling heat transfer in microchannels. Hard to believe, but in this study the scale effects had been addressed without a single mention of the characteristic length of boiling heat transfer. The more so as the real value of the universal characteristic length is of the order of $10^{-5} \mathrm{~m}$ which creates universal context for interpretation and analysis of the entire range of developed boiling heat transfer processes in macro-and microsystems. Without considering this fundamental circumstance, an analysis of the scale effects obviously becomes pointless.

The author's attempt to critically review the work was unsuccessful: in 2011 Editorial Board Int. J. Thermal Sciences went on an unprecedented suppression of scientific debate, rejecting the letter to the editors.

\section{Conclusions}

Long-term confusion with the characteristic length most clearly exhibits the absurd situation in boiling heat transfer research caused by half a century ignoring the basic MTD, the UC and some other boiling fundamentals.

The disregard of the control of heat transfer by the nucleation and corresponding universal characteristic length, average effective radius of nucleation sites (AER), it has led to a thorough deadlock. As a result, it claim to theoretical significance inadequate models and correlations of boiling heat transfer not involving the actual characteristic length. It still remains incomplete standard boiling heat transfer experiment not including investigation of nucleation sites and defining the AER.

Heat transfer community refuses to comply with the main social obligation, permanent expertise and synthesis of research results and ensuring students and professionals on the reliably proven advanced knowledge. The interests of society are sacrificed to a monopoly position of outdated scientific directions and the preservation of opportunity for the infinite development of helpless correlations.

The only exception was made for the problem of boiling heat transfer enhancement resolved in recent decades in direct accordance with the principle established by MPD-UC (just through the universal characteristic length). At the same time, the basic role of the MTD-UC-AER in substantial progress of the relevant $R \& D$ activities passed over in silence in the corresponding publications.

For many years, the author has used all existing scientific means to remedy the existed absurd situation. However, he was unable to overcome enviable unity and coherence of heat transfer community in preventing real scientific debate on the problem.

\section{References}

[1] [Shekriladze, I. G. 1966. "Mechanism of Nucleate Boiling." Bull. Acad. Sci. Georg. SSR. 41: 392-6.

[2] Shekriladze, I. G. and Ratiani, G. V. 1966. "Basic Regularities of Boiling Heat Transfer." Bull. Acad. Sci. Georg. SSR. 42: 145-50.

[3] Shekriladze, I. G. 1967. "Mechanism of Steam Bubble Formation.” NASA-TM-X-59398, RSIC-632, Marshall Space Flight Center, 1-10.

[4] Gorenflo, D., Baumhögger, E., Herres., G. and Kotthoff, S. 2013. "State-of-the-Art of Pool Boiling Prediction Methods." Proc. $4^{\text {th }}$ IIR Conf. Thermophys. Prop. Transf. Proc. Refriger., Paper No TP-004, 1-22.

[5] Shekriladze, I. G. 1981. "Developed Boiling Heat Transfer.” Int. J. Heat Mass Transfer 24: 795-801.

[6] Shekriladze, I. G. 2008. "Boiling Heat Transfer: Mechanisms, Models, Correlations and the Lines of Further Research." The Open Mechanical Engineering Journal 2: 104-27.

[7] Shekriladze, I. G., Gigineishvili, G. I., Rusishvili, J. G. and Shekriladze, D. I. 2010. "Investigation of Duration-Dependent Multifactoring during Boiling on Down-Facing Heating Surface." Proc. of $14^{\text {th }}$ Int. Heat Trans. Conf., IHTC14-23386, 1-10.

[8] Shekriladze, I. G. 2012. "Boiling Heat Transfer: An Overview of Longstanding and New Challenges." $J$. ASTM International 9: 1-41. 
[9] Moore, F. D. and Mesler, R. B. 1961. "The Measurement of Rapid Surface Temperature Fluctuations during Nucleate Boiling of Water." IChE J. 7: 620-4.

[10] Shekriladze, I. G. 1982. "Heat Transfer in Two-Phase Areas with Intensive Evaporation and Condensation." Dr. Tech. Sci. thesis, The Bauman Moscow Highest Technical School.

[11] Shekriladze, I. G. 2014. "Boiling at Macro-and Microscale: Totally Neglected Universal Context." Proc. of 15th FEDSM2014, FEDSM2014-21127, 1-9.

[12] Shekriladze, I. G. 2006. "Developed Boiling Heat Transfer - Forty Years of the Model of 'the Theatre of Director."” Proc. of $13^{\text {th }}$ Int. Heat Trans. Conf. 1-13.

[13] Shekriladze, I. G. 2014. "Boiling Heat Transfer Theory: To Overcome Historical Deadlock." Proc. of $15^{\text {th }}$ Int. Heat Trans. Conf., IHTC15-8817, 1-15.

[14] Jacob, M. 1949. "Heat Transfer." New York: Wiley. 1: 614-52.

[15] Robin, T. T. and Snyder, N. W. 1970. "Bubble Dynamics in Subcooled Nucleate Boiling Based on the Mass Transfer Mechanism.” Int. J. Heat Mass Transfer 13, 305-18.

[16] Nesis, Ye. I. and Sologub, I. S. 1984. "Temperature Fluctuations in Subcooled Boiling at a Single Site." Kipenie i kondensatsia, Riga: RPI Press, 5-13.

[17] Peng, X. F. and Wang, B. X. 1993. "Forced Convection and Flow Boiling Heat Transfer for Liquid Flowing through Microchannels.” Int. J. Heat Mass Transfer 36: 3421-7.

[18] Shoukri, M. and Judd, R. L. 1975. "Nucleation Site Activation in Saturated Boiling." J. Heat Transfer 97: 96-102.

[19] Marto, P. L. and Rohsenow, W. M. 1966. "Effects of Surface Conditions on Nucleate Pool Boiling of Sodium." J. Heat Transf. 88: 149-57.

[20] Chumak, L. V., Malaia, L. V. and Vinichenko, I. V. 1979, "Enhancement of Heat Transfer of Cryogens on the Pipe Surface.” Kholodilnaia Tekhnika, 2: 31-34.

[21] Kruzhilin, G. N. 1948. "Heat Transfer from Heating Surface to Boiling Single-Component Liquid in
Conditions of Natural Convection." Izvestia AN SSSR, OTN 7: 967-80.

[22] Kutateladze, S. S. 1952. "Heat Transfer in Condensation and Boiling." Moscow: Mashgiz Press, 89-94.

[23] Rohsenow, W. M. 1952. "A Method of Correlating Heat Transfer Data for Surface Boiling of Liquids." Trans. ASME 74: 969-76.

[24] Tolubinski, V. I. 1959. "The Problem of Boiling Heat Transfer Theory.” Izvestia VUZ, Energetika, 1: 15-22.

[25] Attinger, D., Frankiewicz, C., Betz, A. R., Ganguly, R., Das, A. and Kim, C. J. et al. 2014. "Surface Engineering for Phase Change Heat Transfer: A Review." MRS Energy \& Sustainability : A Review Journal 1-40.

[26] Pioro, I. L., Rohsenow, W. M. and Doerffer, S. S. 2004. "Nucleate Pool-Boiling Heat Transfer, 1: Review of Parametric Effects of Boiling Surface." Int. J. Heat Mass Transfer 47: 5033-44.

[27] Pioro, I. L., Rohsenow, W. M. and Doerffer, S. S. 2004. "Nucleate Pool-Boiling Heat Transfer, 2: Assessment of Prediction Methods." Int. J. Heat Mass Transfer 47: 5045-57.

[28] Mikic, B. B. and Rohsenow, W. M. 1969. "A New Correlation of Pool-Boiling Data Including the Effect of Heating Surface Characteristics." J. Heat Transfer 91: 245-50.

[29] Griffith, P. and Wallis, S. D. 1960. "The Role of Surface Conditions in Nucleate Boiling." Chem. Engng. Progr. 56: 49-60.

[30] Shekriladze, I. G. and Rusishvili, J. G. 2006. Comments on Papers of L. Pioro, W. Rosenow, and S. Doerfer, "Nucleate Pool-Boiling Heat Transfer, 1: Review of Parametric Effects of Boiling Surface; 2. Assessment of Prediction Methods." Int. J. Heat Mass Transfer 49: 1206-7.

[31] Kim, J. 2009. "Review of Nucleate Pool Boiling Bubble Heat Transfer Mechanisms." Int J. Multiphase Flow. 35: 1067-76.

[32] Kandlikar, S. G. 2010. "Scale Effects on Flow Boiling Heat Transfer in Microchannels: A Fundamental Perspective." Int. J. Thermal Sciences 49: 1073-85. 